\title{
Catechins in neuroAIDS
}

\author{
Italo Mocchetti
}

Received: 30 July 2012 /Revised: 7 August 2012 / Accepted: 12 August 2012 /Published online: 13 September 2012

(C) Journal of NeuroVirology, Inc. 2012

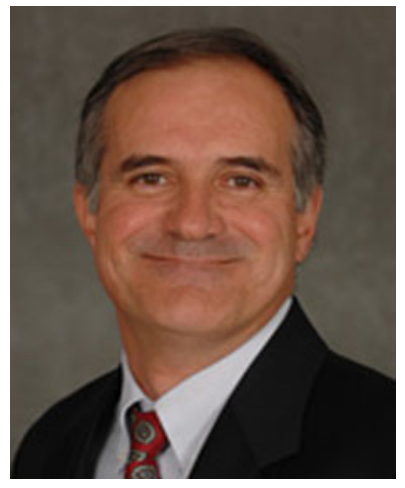

The introduction of highly active antiretroviral therapy (HAART) against human immunodeficiency virus type-1 (HIV) has improved immune recovery as well as decreased the gravity of neurological signs associated with HIV infection of the central nervous system (CNS) (Ellis et al. 2007). Yet, HAART has not eliminated mild neurocognitive deficits and asymptomatic neurocognitive impairments in AIDS patients (McArthur et al. 2003). Thus, the need of adjunct therapies and new strategies that target specifically HIV-mediated pathology in the CNS is great.

The neuropathology of HIV includes neuronal loss as well as reduction of neuronal connectivity, a phenomenon often referred to as synaptic simplification (Ellis et al. 2007), in which both axons and dendrites undergo retraction. While many compounds have been developed to control viral infection, relatively fewer studies have evaluated the ability of drugs to alleviate or prevent the neuropathology of HIV. This

I. Mocchetti $(\bowtie)$

Laboratory of Preclinical Neurobiology,

Department of Neuroscience,

Georgetown University Medical Center,

3970 Reservoir Rd, NW,

Washington, DC 20057, USA

e-mail: moccheti@georgetown.edu is not due to lack of experimental models because the neuropathology observed in humans can be replicated in several animal models of neuroAIDS. Nor does the challenge pertain to the knowledge of pathways of HIV neurotoxicity. Indeed, it has been recognized that HIV, through indirect or direct mechanisms, causes oxidative stress better known as an excessive production of reactive oxygen species (ROS) by the mitochondria. ROS, in turn, can cause extensive cellular injury including a disruption in mitochondrial respiration, protein aggregation, lipid peroxidation, and DNA damage that can lead to cell death by either necrosis or apoptosis (Mattson et al. 2008). Interestingly, oxidative stress is also the main pathway responsible for the majority of neurodegenerative diseases occurring in adults. Pathological states associated with oxidative stress are also triggered by HIV proteins such as the envelope protein gp120 and the transactivation of transcription, or Tat. This is why, as described in this issue of Journal of Neurovirology, Steiners' group has used an in vitro model of oxidative stress to develop a high-throughput screening assay to search for novel compounds that prevent and/or significantly reduce the neurodegenerative process initiated by oxidative stress.

Using rodent and human neuronal cultures, the authors were able to screen a library of 2,000 compounds of naturally occurring substances either approved by the FDA or already tested for safety in humans. Among these, they identified at least two compounds that exhibit neuroprotection against Tat and gp120, including epicatechin and epigallocatechin gallate. These catechins are natural antioxidants that belong to the flavonoids, a family of polyphenolic compounds that are found in plants, fruits (including cocoa), and vegetables, as well as in drinks derived from such plants (e.g., tea, red wine). Remarkably, the authors discovered that the neuroprotective activity of catechins extends beyond their ability to reduce oxidative stress. In fact, they found that catechins prevent Tat-mediated neurite 
damage, one of the hallmarks of neurological dysfunction observed in HIV subjects. Thus, it appears that catechins behave like neurotrophic factors. Is this effect related to their antioxidant properties? To address this question, the authors measured the neurotrophic factor environment in neurons exposed to catechins and observed that these compounds promote an upregulation of the neurotrophin brainderived neurotrophic factor (BDNF). The results are surprising as BDNF is important to many forms of plasticity in relation to chronic neurological conditions which are characterized by loss of neuronal populations especially in the cerebral cortex and basal ganglia (Zuccato and Cattaneo 2009). Remarkably, catechins induce a concomitant decrease in the levels of proBDNF, the larger glycosylated precursor of BDNF that has pro-apoptotic properties. On the other hand, Tat exhibited an opposite effect. Overall, these data suggest that HIV proteins cause a reduction in the activation of the neurotrophin signaling pathway that is necessary not only for the normal maintenance of neurons but also for preventing a disease process that leads to a pathological condition. Thus, catechins, by increasing $\mathrm{BDNF}$ and decreasing proBDNF, reduce the risk of HIV-mediated neuronal degeneration. Although the data generated in this paper were obtained in vitro, their significance cannot be de-emphasized. In fact, the importance of these experimental findings can be appreciated if we consider that BDNF and proBDNF are decreased and increased, respectively, in postmortem brain of HIV subjects with neurocognitive impairment (Bachis et al. 2012).

In light of these new results suggesting that some flavonoids not only prevent HIV-mediated oxidative stress but also may restore the loss of synapses, the next issues to be addressed will almost certainly be whether catechins can be used as an adjunct therapy in HIV subjects. Catechins and other flavonoids are naturally occurring compounds that have been used safely in humans mostly to reduce cardiovascular diseases and as antineoplastic agents. Only a weak estrogenic activity has been reported. Numerous flavonoids, including catechins, can be absorbed after oral administration and pass the blood-brain barrier to reach the CNS. Here, they can interact with $\mathrm{GABA}_{\mathrm{A}}$ receptors and potentiate benzodiazepine action but only at high concentrations. This issue is important because it offers a pharmacological strategy for blocking a pathway significantly involved in neurodegenerative diseases without interfering directly with physiological neurotransmission. A bigger challenge will be to determine when and how catechins should be given to HIV patients.

Overall, this paper represents a clever example of how a research program, initially aimed to search for compounds against neurodegenerative disorders, might be adapted and applied to the field of CNS-HIV infection.

\section{References}

Bachis A, Avdoshina V, Zecca L, Parsadanian M, Mocchetti I (2012) Human immunodeficiency virus type 1 alters brain-derived neurotrophic factor processing in neurons. J Neurosci 32:9477-9484

Ellis R, Langford D, Masliah E (2007) HIV and antiretroviral therapy in the brain: neuronal injury and repair. Nat Rev Neurosci 8:33-44

Mattson MP, Gleichmann M, Cheng A (2008) Mitochondria in neuroplasticity and neurological disorders. Neuron 60:748-766

McArthur JC, Haughey N, Gartner S, Conant K, Pardo C, Nath A, Sacktor N (2003) Human immunodeficiency virus-associated dementia: an evolving disease. J Neurovirol 9:205-221

Zuccato C, Cattaneo E (2009) Brain-derived neurotrophic factor in neurodegenerative diseases. Nat Rev Neurol 5:311-322 